\title{
AiMT
}

Advances in Military Technology

Vol. 15, No. 2, 2020, pp. 265-278

ISSN 1802-2308, eISSN 2533-4123

DOI 10.3849/aimt.01377

\section{Mathematical Modeling of Shooting from Moving Carrier}

\author{
A.M. Lipanov ${ }^{1}$, I.G. Rusyak ${ }^{2}$, V.G. Sufiyanov ${ }^{2 *}$ and S.A. Korolev ${ }^{2}$ \\ ${ }^{1}$ Keldysh Institute of Applied Mathematics, Moscow, Russia, \\ ${ }^{2}$ Kalashnikov Izhevsk State Technical University, Izhevsk, Russia,
}

The manuscript was received on 15 January 2020 and was accepted after revision for publication as research paper on 2 August 2020.

\begin{abstract}
:
The work is devoted to the development of software complex for modeling the dynamic characteristics of a moving carrier in burst firing mode and to the study of their impact on shooting accuracy. A mathematical model for mechanical system of moving carrier is given, which allows to take into account the movement and vibrations of the helicopter, as well as the mutual influence of the system "helicopter - rocket-gun armament". The research results of the dispersion of projectiles and rockets in serial firing, depending on the parameters of the movement of moving carrier and the shooting mode, are presented. The paper gives a description and outlines working principles of the software complex for visualization of simulation results.
\end{abstract}

\section{Keywords:}

burst fire, dispersion of projectiles and rockets, external ballistics, mathematical modeling, mechanical system, moving carrier

\section{Introduction}

The development of computer technologies allows creating visualization systems for training in actions close to reality as a preparation for combat operations and, in particular, for three-dimensional virtual simulation of combat operations on land, air and water (see, for example, [1-3]).

This paper presents the results of the development of a software complex for simulating the firing of rocket-gun armament from a moving carrier. To solve this problem, a mathematical model of the "moving carrier - rocket-gun armament" system was developed.

\footnotetext{
* Corresponding author: Kalashnikov Izhevsk State Technical University, Studencheskaya 7, Izhevsk, Russian Federation. Phone: +7 (3412) 7760 55, Fax: +7 (3412) 5040 55, E-mail: vsufiy@mail.ru
} 
When serial firing is conducted from a moving carrier, it is required to determine the trajectory of the projectiles and rockets, taking into account the interaction of the moving carrier and weapons which affects the shooting accuracy: transport vibrations created by screws and turbines, and vibrations caused by shooting ammunition.

\section{Mathematical Model of Mechanical System "Helicopter - Rocket-Gun Armament"}

Mechanical system "helicopter-armament" will be presented in the form of a set $K$ of material points with masses $m_{k}, k=\overline{0, K}$. Three types of material points are considered [4]:

- rigid shell $(\mathrm{k}=0)$ without moving parts (helicopter body),

- moving material points of carrier $\left(k=\overline{1, K_{1}}\right)$, the movement of which leads to vibrations (screws and turbines),

- moving material points $\left(k=\overline{K_{1}+1, K}\right)$ that can leave the system (projectiles and rockets), where $K=K_{1}+K_{s}+K_{r}, K_{s}$ is the number of projectiles, $K_{r}$ is the number of rockets.

The dynamics of the mechanical system is considered in two coordinate systems: a fixed (inertial) system $(O x y z)$ and a moving one $\left(O_{1} \xi \eta \varsigma\right)$ rigidly connected with the body (Fig. 1).

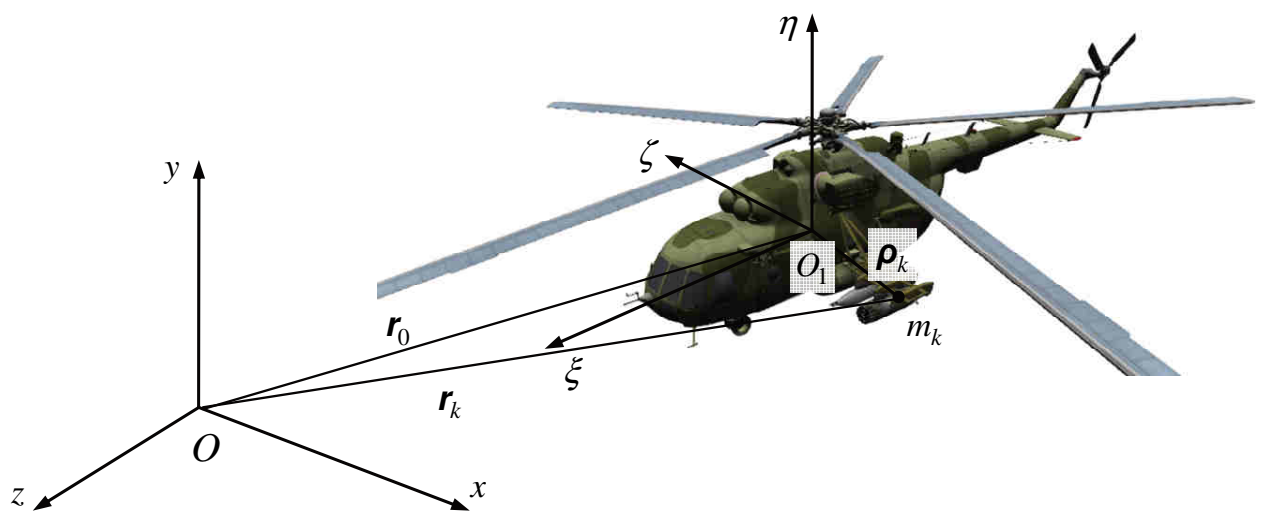

Fig. 1 Scheme of the mechanical system "helicopter-rocket-gun armament"

The center of the moving coordinate system (point $O_{1}$ ) coincides with the mass center of the rigid shell and has coordinates in a fixed coordinate system $\boldsymbol{r}_{0}$.

The radius-vector of a material point in a fixed coordinate system $O x y z$ is denoted as $\boldsymbol{r}_{k}=\left(x_{k}, y_{k}, z_{k}\right)^{\mathrm{T}}$, and in a moving coordinate system $O_{1} \xi \eta \varsigma$ as $\boldsymbol{\rho}_{k}=\left(\xi_{k}, \eta_{k}, \varsigma_{k}\right)^{\mathrm{T}}$.

To derive equations describing the movement of points of a mechanical system, the kinetic energy of the system under consideration is written in the form $[5,6]$ :

$$
T=\frac{1}{2} \sum_{k=0}^{K} m_{k}\left(\frac{\mathrm{d} \boldsymbol{r}_{k}}{\mathrm{~d} t} \cdot \frac{\mathrm{d} \boldsymbol{r}_{k}}{\mathrm{~d} t}\right)
$$


The movement velocity of material points in a fixed coordinate system is determined by the expression:

$$
\frac{\mathrm{d} \boldsymbol{r}_{k}}{\mathrm{~d} t}=\boldsymbol{V}+\dot{\boldsymbol{\rho}}_{k}+\left[\boldsymbol{\omega} \times \boldsymbol{\rho}_{k}\right]
$$

where $\boldsymbol{V}=\mathrm{d} \boldsymbol{r}_{0} / \mathrm{d} t$ is the velocity of moving coordinate system origin; $\boldsymbol{\omega}$ is the angular velocity of rotation of the moving coordinate system.

The impulse of the mechanical system $\boldsymbol{P}=\partial T / \partial \boldsymbol{V}$ with regard to Eqs (1) and (2) is calculated by the formula:

$$
\boldsymbol{P}=m \boldsymbol{V}+\sum_{k=1}^{K} m_{k} \dot{\boldsymbol{\rho}}_{k}+\sum_{k=1}^{K} m_{k}\left[\boldsymbol{\omega} \times \boldsymbol{\rho}_{k}\right]
$$

where $m=m_{0}+\sum_{k=1}^{K} m_{k}$ is the total mass of the mechanical system.

The kinetic moment of the system is determined by the expression $\boldsymbol{M}=\partial T / \partial \boldsymbol{\omega}$, from where, taking into account Eqs (1) and (2), the following is obtained:

$$
\boldsymbol{M}=\boldsymbol{B} \boldsymbol{\omega}+\sum_{k=1}^{K} m_{k}\left\{\boldsymbol{\rho}_{k} \times\left[\boldsymbol{\omega} \times \boldsymbol{\rho}_{k}\right]\right\}+\sum_{k=1}^{K} m_{k}\left[\boldsymbol{\rho}_{k} \times \dot{\boldsymbol{\rho}}_{k}\right]+\left[\sum_{k=1}^{K} m_{k} \boldsymbol{\rho}_{k} \times \boldsymbol{V}\right]
$$

where $\boldsymbol{B}=\left(\begin{array}{ccc}J_{\xi} & -D_{\varsigma} & -D_{\eta} \\ -D_{\varsigma} & J_{\eta} & -D_{\xi} \\ -D_{\eta} & -D_{\xi} & J_{\varsigma}\end{array}\right)$ is the matrix of inertia moments of a rigid shell; $\mathbf{J}_{\xi}, J_{\eta}$, $J_{\varsigma}, D_{\xi}, D_{\eta}, D_{\varsigma}$ are axial and centrifugal moments of inertia of a rigid shell, calculated by the formulas:

$$
\begin{array}{ll}
J_{\xi}=\int\left(\eta^{2}+\varsigma^{2}\right) \mathrm{d} m, & D_{\xi}=\int \eta \varsigma \mathrm{d} m \\
J_{\eta}=\int\left(\xi^{2}+\varsigma^{2}\right) \mathrm{d} m, & D_{\eta}=\int \xi \varsigma \mathrm{d} m \\
J_{\varsigma}=\int\left(\eta^{2}+\xi^{2}\right) \mathrm{d} m, & D_{\varsigma}=\int \xi \eta \mathrm{d} m
\end{array}
$$

Translational $\boldsymbol{V}$ and angular $\boldsymbol{\omega}$ velocities of the moving carrier are determined from a system

$$
\boldsymbol{A} \boldsymbol{W}=\boldsymbol{b}
$$

where $\boldsymbol{W}=(\boldsymbol{V}, \boldsymbol{\omega})^{\mathrm{T}}=\left(v_{1}, v_{2}, v_{3}, \omega_{1}, \omega_{2}, \omega_{3}\right)^{\mathrm{T}}$. The matrix $\boldsymbol{A}$ and the vector $\boldsymbol{b}$ are determined in accordance with Eqs (3) and (4).

To determine the position of the moving coordinate system origin $O_{1}=\left(x_{0}, y_{0}, z_{0}\right)$ and orientation angles of the helicopter, the system of equations [4] is solved:

$$
\begin{aligned}
& \frac{\mathrm{d} x_{0}}{\mathrm{~d} t}=(\boldsymbol{V} \cdot \boldsymbol{\alpha}), \frac{\mathrm{d} y_{0}}{\mathrm{~d} t}=(\boldsymbol{V} \cdot \boldsymbol{\beta}), \frac{\mathrm{d} z_{0}}{\mathrm{~d} t}=(\boldsymbol{V} \cdot \boldsymbol{\gamma}) \\
& \frac{\mathrm{d} \boldsymbol{\alpha}}{\mathrm{d} t}=[\boldsymbol{\alpha} \times \boldsymbol{\omega}], \frac{\mathrm{d} \boldsymbol{\beta}}{\mathrm{d} t}=[\boldsymbol{\beta} \times \boldsymbol{\omega}], \frac{\mathrm{d} \boldsymbol{\gamma}}{\mathrm{d} t}=[\boldsymbol{\gamma} \times \boldsymbol{\omega}]
\end{aligned}
$$

where $\boldsymbol{\alpha}=\left(\begin{array}{l}\alpha_{1} \\ \alpha_{2} \\ \alpha_{3}\end{array}\right), \boldsymbol{\beta}=\left(\begin{array}{l}\beta_{1} \\ \beta_{2} \\ \beta_{3}\end{array}\right), \boldsymbol{\gamma}=\left(\begin{array}{l}\gamma_{1} \\ \gamma_{2} \\ \gamma_{3}\end{array}\right)$ are direction cosines forming the transition matrix $\boldsymbol{Q}=(\boldsymbol{\alpha}, \boldsymbol{\beta}, \boldsymbol{\gamma})$ from the fixed $O x y z$ to the moving $O_{1} \xi \eta \varsigma$ coordinate system. 
The equations of the change of impulse and moment in the moving coordinate system are written in the form:

$$
\begin{gathered}
\frac{\mathrm{d} \boldsymbol{P}}{\mathrm{d} t}+[\boldsymbol{\omega} \times \boldsymbol{P}]=\boldsymbol{Q} \boldsymbol{F}_{0} \\
\frac{\mathrm{d} \boldsymbol{M}}{\mathrm{d} t}+[\boldsymbol{\omega} \times \boldsymbol{M}]+[\boldsymbol{V} \times \boldsymbol{P}]=\boldsymbol{M}_{0}
\end{gathered}
$$

where $\boldsymbol{F}_{0}, \boldsymbol{M}_{0}$ are external force and moment acting on the mechanical system.

The mechanical system under consideration is affected by the force of gravity, the resistance force and the thrust force of the helicopter. We assume that at the initial moment of time, the thrust force balances the force of gravity and the resistance force, that is, the helicopter is in the hover mode, or moves at a constant speed. Each moving point is affected by gravity $\boldsymbol{F}_{k}=\left(0,-m_{k} g, 0\right)^{\mathrm{T}}$. For the interior points $\sum_{k=1}^{K(t)} \boldsymbol{F}_{k} \neq 0$, the resultant force applied to the rigid shell in the fixed coordinate system is

$$
\boldsymbol{F}_{0}=\left(0, \sum_{k=K(t)}^{K} m_{k} g, 0\right)^{\mathrm{T}}
$$

where $K(t)$ is the number of material points in the system, which changes with time during the shooting of ammunition.

The moment of external forces acting on the mechanical system is determined by the formula

$$
\boldsymbol{M}_{0}=\left[\sum_{k=0}^{K(t)} \boldsymbol{\rho}_{k} \times \boldsymbol{Q} \boldsymbol{F}_{k}\right]
$$

The initial conditions for the system of differential Eqs (7) and (8) are determined from Eqs (3) and (4) for the initial coordinates and velocities of the points of the mechanical system at $t=0$.

The initial conditions for the vectors $\boldsymbol{\alpha}, \boldsymbol{\beta}, \boldsymbol{\gamma}$ are defined through the angles that determine the position of the helicopter in space: $\varphi$ is the roll angle; $\psi$ is the yaw angle; $\theta$ is the pitch angle:

$$
\boldsymbol{Q}^{\mathrm{T}}=(\boldsymbol{\alpha}, \boldsymbol{\beta}, \boldsymbol{\gamma})^{\mathrm{T}}=\boldsymbol{G}(\phi, \psi, \theta)=\boldsymbol{G}_{2}(\psi) \boldsymbol{G}_{3}(\theta) \boldsymbol{G}_{1}(\phi),
$$

where $\boldsymbol{G}_{1}(\varphi), \boldsymbol{G}_{2}(\psi), \boldsymbol{G}_{3}(\theta)$ are rotation matrices at the angles of $\varphi, \psi, \theta$, respectively.

The angles $\varphi, \psi, \theta$ in the calculation process are expressed through the direction cosines using the following formulas:

$$
\varphi=-\frac{\arcsin \beta_{3}}{\sqrt{1-\beta_{1}^{2}}}, \psi=-\frac{\arcsin \gamma_{1}}{\sqrt{1-\beta_{1}^{2}}}, \theta=\arcsin \beta_{1}
$$

For the sources of vibrations (screws and turbines), the characteristics $\boldsymbol{\rho}_{k}(t), \dot{\boldsymbol{\rho}}_{k}(t), k=\overline{1, K_{1}}$ are given in the form:

$$
\begin{aligned}
& \boldsymbol{\rho}_{k}(t)=\boldsymbol{\rho}_{k}(0)+\boldsymbol{L}_{k} \sin \left(2 \pi \Omega_{k} t\right) \\
& \dot{\boldsymbol{\rho}}_{k}(t)=\boldsymbol{L}_{k} 2 \pi \Omega_{k} \cos \left(2 \pi \Omega_{k} t\right)
\end{aligned}
$$


where $\boldsymbol{L}_{k}$ is the vibration amplitude vector and $\boldsymbol{\Omega}_{k}$ is the vibration frequency vector, which are determined on the basis of experimental amplitude-frequency vibration indicators at various points of the rigid shell.

For the material points corresponding to the ammunition, the characteristics $\boldsymbol{\rho}_{k}(t), \dot{\boldsymbol{\rho}}_{k}(t), k=\overline{K_{1}+1, K}$ are determined by the laws of the projectile movement in the barrel during the shot and the movement of the rocket along the directing launcher during the launch.

The system of differential Eqs of motion (6)-(8) is solved numerically by the Runge-Kutta-Verner method of the $6^{\text {th }}$ order of accuracy with control of the integration error [7]. The algebraic system (5) is solved at each iteration in time by inverting the matrix $\boldsymbol{A}$.

\section{Calculation of Initial Shooting Conditions and Trajectories of Projectiles and Rockets}

The initial shooting conditions at the moments of shots $t_{k}^{0}, k=\overline{K_{1}+1, K}$ are calculated according to the results of modeling the mechanical system of moving carrier [8].

The trajectory of the projectile is built in a fixed starting coordinate system $O_{\mathrm{c}} x_{\mathrm{c}} y_{\mathrm{c}} z_{\mathrm{c}}$ associated with the mass center of the projectile at the moment of the shot $\boldsymbol{r}_{k}^{0}=\boldsymbol{r}_{k}\left(t_{k}^{0}\right)$, where the axis $x_{\mathrm{c}}$ is directed along the directional shooting angle $\alpha_{d}$ in the horizontal plane, the axis $y_{\mathrm{c}}$ is directed vertically, the axis $z_{\mathrm{c}}$ forms the right three vectors (Fig. 2).

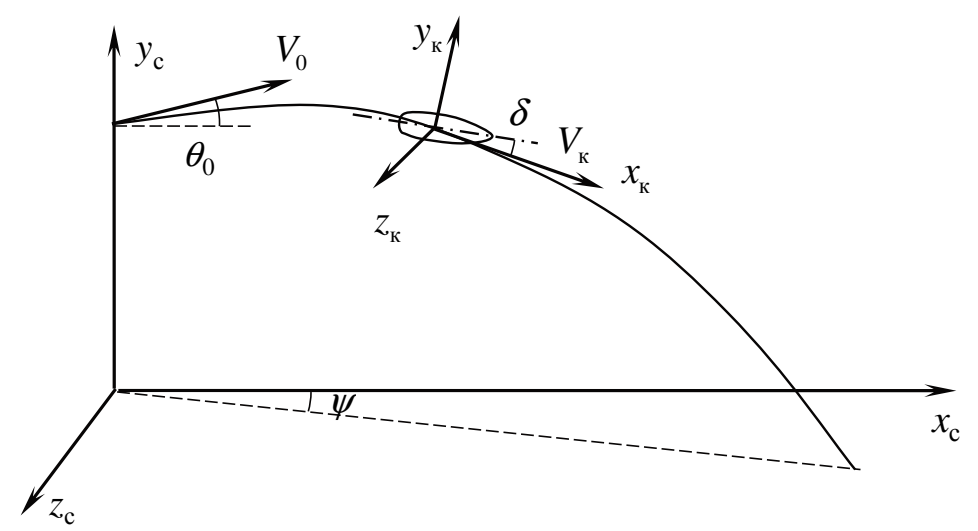

Fig. 2 Projectile motion trajectory

The initial velocity of the projectile in the starting coordinate system is determined from (2):

$$
\boldsymbol{V}_{k}^{0}=\frac{\mathrm{d} \boldsymbol{r}_{k}\left(t_{k}^{0}\right)}{\mathrm{d} t}=\left(V_{x k}^{0}, V_{y k}^{0}, V_{z k}^{0}\right)^{\mathrm{T}}
$$

where $V_{x k}^{0}, V_{y k}^{0}, V_{z k}^{0}$ are projections of the velocity vector on the coordinate axes $x_{\mathrm{c}}, y_{\mathrm{c}}$, $z_{\mathrm{c}}$ for the $k^{\text {th }}$ projectile at the moment of the shot. 
The initial conditions for solving the problem of external ballistics are the modulus of the initial velocity of the projectile, taking into account the movement of the moving carrier,

$$
V_{k}^{0}=\sqrt{\left(V_{x k}^{0}\right)^{2}+\left(V_{y k}^{0}\right)^{2}+\left(V_{z k}^{0}\right)^{2}}
$$

and the initial values of the inclination angle of the trajectory $\theta$ and the direction angle of $\operatorname{shot} \psi$,

$$
\theta_{k}^{0}=\arctan \frac{V_{y k}^{0}}{V_{x k}^{0}}, \psi_{k}^{0}=\arctan \frac{V_{z k}^{0}}{V_{x k}^{0}}
$$

Since the axis of the gun and, accordingly, the symmetry axis of the projectile do not coincide with the total velocity vector of the projectile during the motion of the moving carrier, nutation angles arise, which are equal at the initial time:

$$
\delta_{1 k}^{0}=\psi_{k}^{m c}-\psi_{k}^{0}, \delta_{2 k}^{0}=\theta_{k}^{m c}-\theta_{k}^{0}
$$

where $\psi_{k}^{m c}=\psi\left(t_{k}^{0}\right), \theta_{k}^{m c}=\theta\left(t_{k}^{0}\right)$ are the orientation angles of the moving carrier in space at the shots moments (we assume that the direction of the weapon or launcher coincides with the direction of the longitudinal axis of the helicopter), defined by Eq. (9).

The initial values of the horizontal and vertical components of the equatorial angular velocity of the projectile are determined through the components of the angular velocity of the moving carrier:

$$
\omega_{1 k}^{0}=\omega_{y}^{m c}\left(t_{k}^{0}\right), \omega_{2 k}^{0}=\omega_{z}^{m c}\left(t_{k}^{0}\right)
$$

The motion trajectory of the projectiles and rockets is calculated based on the method for solving the problem of external ballistics when shooting from a moving carrier, presented in works [8,9], under initial conditions defined by Eqs (10)-(13).

To calculate the coefficients of aerodynamic forces and moments acting on the projectile, numerical technique for simulation of flow past a projectile along the trajectory is implemented, based on the numerical solution of Favre-averaged Navier-Stokes equations (FANS) of continuum motion using the $k-\varepsilon$ turbulence model [10]. According to the results of the numerical experiment, using the least squares method, regression dependencies were constructed for the coefficients of aerodynamic forces and moments for different Mach numbers, angles of attack, and dimensionless rotational velocities of the projectile [11].

Approximation dependences for the coefficients of aerodynamic force, taking into account the significance analysis of the coefficients of the regression equations, have the form:

$$
\begin{aligned}
& C_{x}^{\mathrm{a}}(M, \alpha)=a_{x 0}+a_{x 1} M+a_{x 2} M^{2}+a_{x 3} M^{3}+a_{x 4} \alpha^{2} \\
& C_{y}^{\mathrm{a}}(M, \alpha)=a_{y 0}+a_{y 1} \alpha+a_{y 2} \alpha^{3}+a_{y 3} M \alpha+a_{y 4} M^{2} \alpha \\
& C_{z}^{\mathrm{a}}\left(\bar{\omega}_{x}, \alpha\right)=a_{z 0}+a_{z 1} \alpha+a_{z 2} \alpha^{2}+a_{z 3} \bar{\omega}_{x} \alpha+a_{z 4} \bar{\omega}_{x} \alpha^{2}
\end{aligned}
$$

The corresponding approximation dependences for the moment coefficients are:

$$
\begin{aligned}
m_{x}^{\mathrm{a}}\left(M, \bar{\omega}_{x}\right) & =\left(b_{x 0}+b_{x 1} M+b_{x 2} M^{2}\right) \bar{\omega}_{x} \\
m_{y}^{\mathrm{a}}\left(M, \alpha, \bar{\omega}_{x}\right) & =\left(b_{y 0}+b_{y 1} M+b_{y 2} M^{2}\right)\left(\alpha+b_{y 3} \alpha^{2}\right) \bar{\omega}_{x} \\
m_{z}^{\mathrm{a}}(M, \alpha) & =\left(b_{z 0}+b_{z 1} M+b_{z 2} M^{2}\right) \alpha
\end{aligned}
$$


Here, $a_{x i}, a_{y i}, a_{z i}, b_{x i}, b_{y i}, b_{z i}$ are the coefficients of the regression equations. Approximation error of the calculated data does not exceed $1 \%$.

\section{Simulation of Influence of Disturbing Factors on Motion Trajectories of Projectiles and Rockets}

To take into consideration the effect of the helicopter rotor on the movement of projectiles and rockets in the initial part of the trajectory, a method has been developed for calculating the parameters of the air flow generated by the screw rotation [12]. For a given flight mode of a helicopter, the average value of the inductive velocity $\boldsymbol{v}^{m r}=\left(v_{\xi}^{m r}, v_{\eta}^{m r}, v_{\zeta}^{m r}\right)$ is determined on the basis of solving the equations of the stationary motion of the helicopter. The longitudinal and lateral velocity components $v_{\xi}^{m r}, v_{\zeta}^{m r}$ are considered constant in the main rotor plane. The distribution of the normal component of the inductive velocity $v_{\eta}^{m r}$ is set as a combination of two specific types of distributions shown in Fig. 3 [13]:

$$
v_{\eta}^{m r}(r, \varphi)=\bar{v}_{\eta}^{m r}\left[\sqrt{1,5} \bar{r}^{1 / 2} f_{1}(\mu)+(1+\bar{r} \cos \varphi) f_{2}(\mu)\right]
$$

where $r, \phi$ are radius and azimuth ( $\phi=0$ corresponds to the direction opposite to the helicopter movement) of a point in the plane of the screw; $\bar{v}_{\eta}^{m r}$ is the average inductive velocity; $\bar{r}=r / R_{m r}$ is the dimensionless radius; $R_{m r}$ is the radius of the main rotor; $\mu=V_{m c} /\left(\omega_{m r} R_{m r}\right)$ is the dimensionless flight mode parameter; $V_{m c}$ is the helicopter flight velocity; $\omega_{m r}$ is the screw rotation velocity; $f_{1}(\mu), f_{2}(\mu)$ are empirical functions, $f_{1}(\mu)+f_{2}(\mu)=1$.

For the salvo launches from blocks of rockets, one of the factors affecting the shooting accuracy is the variation caused by the influence of rockets on each other. So the distance between the corps of the nearest rockets $l$ can be from $0.8 \mathrm{~m}$ to $1.2 \mathrm{~m}$. Therefore, subsequent rockets in the bursts are moving in the aerodynamic trail of previous rockets. When solving the trajectory problem, it is necessary to take this factor into account as corrections of the aerodynamic coefficients calculated in an unperturbed medium. The scheme of orientation of the rocket relative to the jet stream is shown in Fig. 4.

The simulation of a jet engine flow around a rocket is based on the numerical solution of the Favre-averaged Navier-Stokes equations in the ANSYS Fluent software package. The approximation dependences for corrections of the aerodynamic coefficients of forces and moments when a rocket moves in a jet stream have the following form [14]:

$$
\begin{aligned}
\Delta C_{x}^{j s}(\bar{l}, \bar{r}, \delta) & =a_{x 0} \bar{l}^{a_{x 1}}\left(1-\bar{r}^{3 / 2}\right)^{a_{x 2}}\left(1+\bar{r}^{1 / 2} \delta^{2}\right) \\
\Delta C_{y}^{j s}\left(\bar{l}, \bar{r}, \delta_{r}\right) & =a_{y 0}^{+/-} \bar{l}^{a_{y 1}}\left(1-\bar{r}^{3 / 2}\right)^{a_{y 2}} \delta_{r} \\
\Delta C_{z}^{j s}\left(\bar{l}, \bar{r}, \delta_{t}\right) & =a_{z 0} \bar{l}^{a_{z 1}}\left(1-\bar{r}^{3 / 2}\right)^{a_{z 2}} \delta_{t} \\
\Delta m_{x}^{j s}(\bar{l}, \bar{r}) & =b_{x 0} \bar{l}^{b_{x 1}}\left(1-\bar{r}^{3 / 2}\right)^{b_{x 2}} \\
\Delta m_{y}^{j s}\left(\bar{l}, \bar{r}, \delta_{t}\right) & =b_{y 0} \bar{l}^{b_{y 1}}\left(1-\bar{r}^{3 / 2}\right)^{b_{y 2}} \delta_{t} \\
\Delta m_{z}^{j s}\left(\bar{l}, \bar{r}, \delta_{r}\right) & =b_{z 0}^{+/-} \bar{l}^{b_{z 1}}\left(1-\bar{r}^{3 / 2}\right)^{b_{z 2}} \delta_{r}
\end{aligned}
$$


where $\bar{l}=l / L_{j s}$ is the dimensionless coordinate along the length of the jet; $L_{j s}$ is the length of the considered section of the jet; $\bar{r}=r / R_{j s}(l)$ is the dimensionless coordinate along the jet radius; $R_{j s}(l)$ is the jet radius; $a_{x i}, a_{y i}, a_{z i}, b_{x i}, b_{y i}, b_{z i}$ are coefficients of regression equations. The approximation error for all dependencies does not exceed $3 \%$.

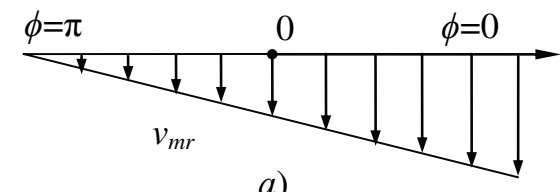

a)

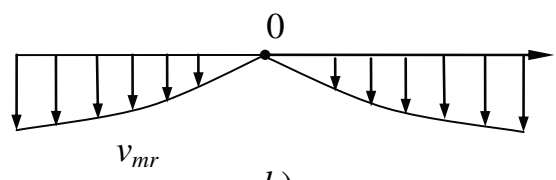

b)

Fig. 3 Distribution shapes of inductive velocity of helicopter rotor: a) with vertical flight mode; $b$ ) with maximum horizontal flight speed.

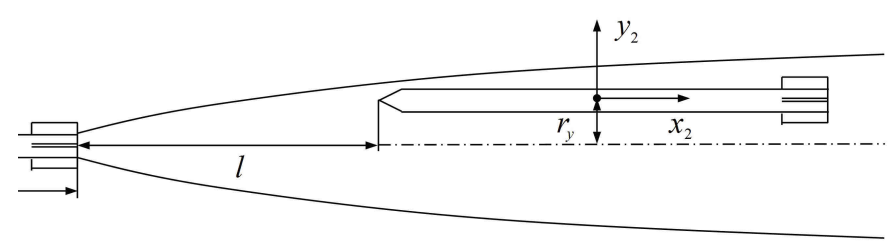

a)

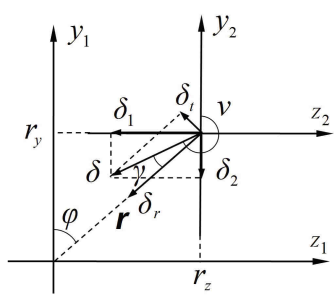

b)

Fig. 4 Scheme of rocket orientation relative to jet stream:

a) side view; b) front view

\section{Software Complex for Modeling and Visualization of Shooting Results from Moving Carrier}

The software complex for simulation and visualization of shooting of rocket-gun armament from a moving carrier was created in MS Visual Studio 2013 in C\# using the NeoAxis 3.5 SDK [15]. The basis of the program complex is the information core which contains a database and a database administration system. The database of the software complex comprises the information about moving carriers, rocket-gun armament, projectiles and rockets, and infrastructure facilities; the digital map of the area; the virtual geometric models of modeling objects; and the results of mathematical modeling and field experiments. The information in the software package is stored in XML format, which provides flexibility in the formation of the data structure.

The software complex consists of the following blocks: modeling the mechanical system of a moving carrier; modeling projectiles and rockets from a moving carrier; modeling of probabilistic characteristics of target destruction and a block of visualization of simulation results.

The block for modeling of a mechanical system enables calculating and plotting dynamic and kinematic characteristics of a mechanical model of a moving carrier. The block for modeling the movement of projectiles and rockets from a moving carrier allows the calculation and plotting of trajectories taking into account the influence of 
disturbing factors on the initial conditions of movement of projectiles and rockets. The hydrodynamic model of the moving carrier allows to take into account the effect of the air flow from the main rotor in the initial part of the trajectory of the projectiles and rockets. The hydrodynamic model of the flow around a rocket makes it possible to consider the effect of the impact of a jet engine stream ahead of a flying rocket.

The simulation methodology for modeling the probabilistic characteristics of hitting a target takes into consideration the laws of the distribution of factors affecting dispersion and allows the method of statistical tests to determine the spread of the incidence points of projectiles and rockets and the probability characteristics of hitting the target (Fig. 5).

The block for visualization creates conditions for importing simulation results of shooting tests from a moving carrier. The trajectories are calculated in the starting coordinate system and converted into the earth coordinate system. On the basis of these data, it is possible to observe the dynamics of the movement of three-dimensional models of a moving carrier, launching projectiles and rockets along a calculated trajectory and hitting a target on a digital map of the area (Fig. 6).

The incidence points of projectiles and rockets are displayed taking into account the real terrain. The simulation results allow to optimize the target situation and determine the time and position of the moving carrier at shot in dynamics, to determine the exact positioning coordinates and parameters of the functioning of the measuring equipment for registering the trajectories of projectiles and rockets.

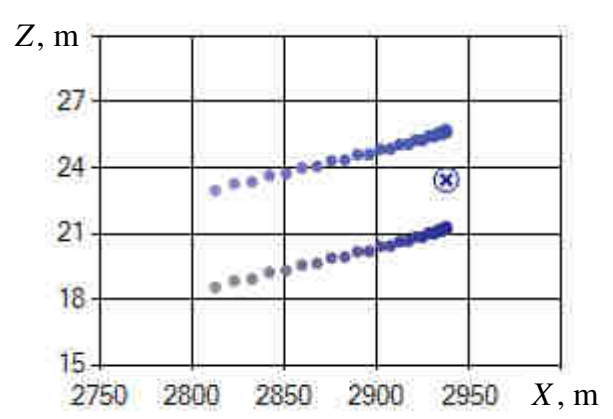

a)

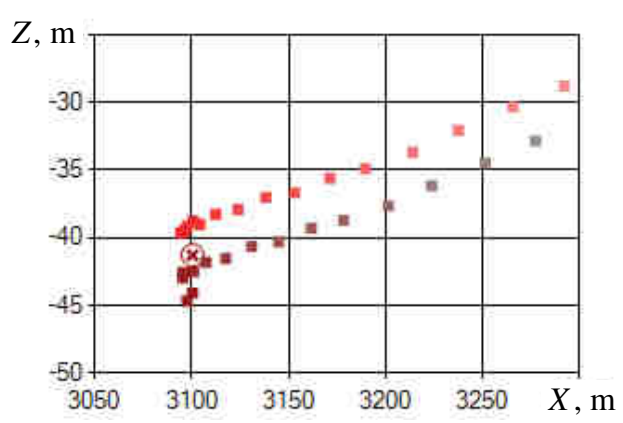

b)

Fig. 5 The incidence points of projectiles and rockets when fire in bursts: a) projectiles; b) rockets

\section{Simulation Results of Hooting from a Moving Carrier}

A military-transport helicopter Mi-8 is considered as a movement carrier with weapons [14]:

- the aircraft gun of $23 \mathrm{~mm}$ caliber with a high explosive-fragmentationincendiary projectile OFZ (interval between shots is $\tau_{s}=0.02 \mathrm{~s}$, the projectile mass is $m_{s}=0.18 \mathrm{~kg}$, the initial velocity is $V_{s 0}=740 \mathrm{~m} \cdot \mathrm{s}^{-1}$ ),

- the launching block of unguided aircraft rockets C-8 with $80 \mathrm{~mm}$ caliber (interval between launches is $\tau_{r}=0.05 \mathrm{~s}$, the rocket mass is $m_{r}=16.3 \mathrm{~kg}$, the initial velocity is $V_{r 0}=42 \mathrm{~m} \cdot \mathrm{s}^{-1}$ ). 


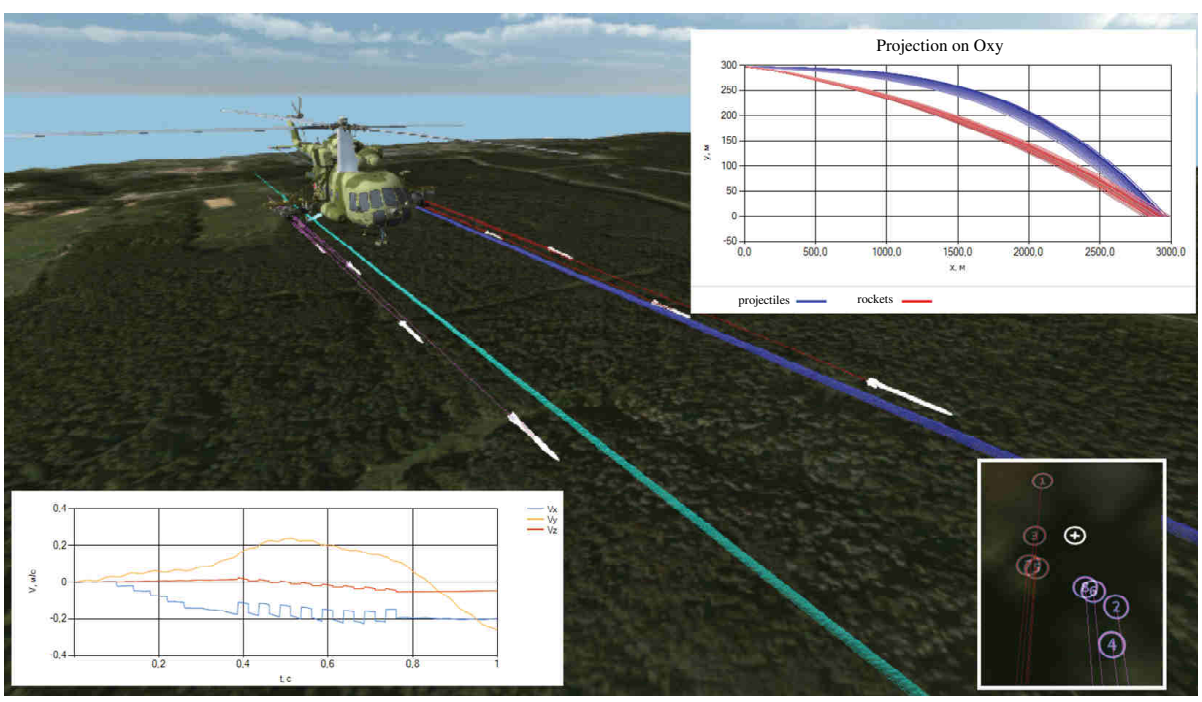

Fig. 6 Motion trajectories of moving carrier, projectiles and rockets

Various shooting modes were considered in the study of the influence of motion parameters of the moving carrier on the trajectory of projectiles and rockets (Tab. 1). The horizontal velocity of the helicopter $V_{m c}$ varied in the range of $50 \mathrm{~km} \cdot \mathrm{h}^{-1}$ to $250 \mathrm{~km} \cdot \mathrm{h}^{-1}$. The average shooting range is $3000 \mathrm{~m}$.

Fig. 7 presents the simulation results for the considered shooting modes at a helicopter speed of $100 \mathrm{~km} \cdot \mathrm{h}^{-1}$. The graphs show the maximum deviations in range $\Delta X$ of the incidence points for the projectiles and rockets in the bursts, depending on the bursts time $\Delta t$.

Tab. 1 Modes of shooting from a moving carrier

\begin{tabular}{|c|l|c|c|}
\hline & \multicolumn{1}{|c|}{ Shooting mode } & $\begin{array}{c}\text { Number of projectiles } \\
\text { [rockets] }\end{array}$ & $\begin{array}{c}\text { Turn time } \Delta t \\
{[\mathrm{~s}]}\end{array}$ \\
\hline 1 & $\begin{array}{l}\text { Shooting from 2 guns in parallel } \\
\text { from each side }\end{array}$ & $20-100$ & $0.2-1.0$ \\
\hline 2 & $\begin{array}{l}\text { Launches from 2 blocks of rockets } \\
\text { alternately from each side }\end{array}$ & $10-40$ & $0.25-1.0$ \\
\hline
\end{tabular}

From the above graphs it can be seen that the deviation of the projectiles in the bursts from the aiming point occurs in the direction of decreasing the range. This is explained by the fact that at shot from guns due to recoil, the pitch angle of the helicopter deviates in the negative direction, therefore, its longitudinal axis leans down. When rockets are launched, the opposite is true. Due to the change in the total impulse of the system "helicopter - armament", the longitudinal axis of the helicopter is deflected upwards. Therefore, the deviation of the rockets in the bursts from the aiming point occurs in the direction of increasing the range. With an increase in the bursts length, the spread of the incidence points of projectiles and rockets is progressively increasing. 


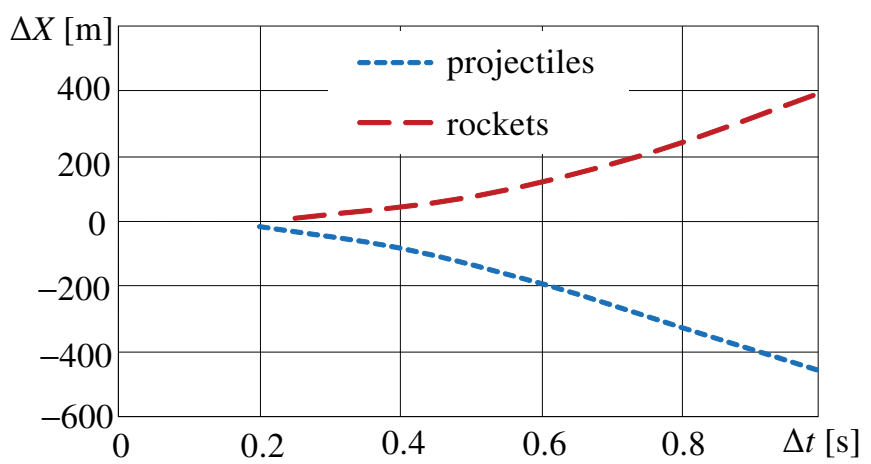

Fig. 7 Dependence of maximum deviation of incidence points of projectiles and rockets in the bursts by distance $\Delta X$ on the bursts time $\Delta t$

Fig. 8 shows the maximum deviations in the range $\Delta X$ of the incidence points of projectiles and rockets in the bursts, depending on the motion speed of the moving carrier $V_{m c}$. Shooting is made from two sides, the number of projectiles in the bursts 50 , the number of rockets -20 .

With an increase in the speed of the moving carrier, the dispersion of projectiles in distance decreases. This is due to the increase in the total velocity of the projectile, which partially compensates for the deviation of the axis of the helicopter down. The rockets spread in range is influenced by two factors: the recoil impulse during launch and the change in the total impulse of the system "helicopter - armament" when unloading the launch blocks. For the helicopter speeds of less than $75 \mathrm{~km} \cdot \mathrm{h}^{-1}$ due to recoil, the rocket bursts deviation occurs in the direction of decreasing range. For higher flight speeds, the prevailing factor is the change in the total impulse of the system and the deviation of the rocket bursts occurs in the direction of increasing the shooting range.

Fig. 9 shows the maximum deviations in range $\Delta X$ of the incidence points of the projectiles and rockets in the bursts, depending on the interval between the shots $\tau$. When the time interval between the shots of projectiles reduces (rocket launches), the accuracy of the bursts increases. However, if the interval between rocket launches decreases to less than $0.02 \mathrm{~s}$, subsequent rockets in the bursts begin to be influenced by the jet engine stream ahead of the flying rockets, therefore the deviation of the rocket incidence points increases dramatically.

One of the factors affecting the accuracy of helicopter shooting is the air flow generated by the main rotor. The air flow from the main rotor of the helicopter has a significant impact on the projectiles movement. Fig. 10 shows the maximum deviations in the range of the incidence points of rockets in the bursts under the action of the air flow from the main rotor, depending on the speed of the helicopter.

The simulation results show that the effect of the main rotor on the trajectory of the rockets is manifested at helicopter speeds of less than $80 \mathrm{~km} \cdot \mathrm{h}^{-1}$. At high speeds of movement, the air stream from the main rotor is carried to the tail end of the helicopter and the rockets do not fall into the area of its influence. 


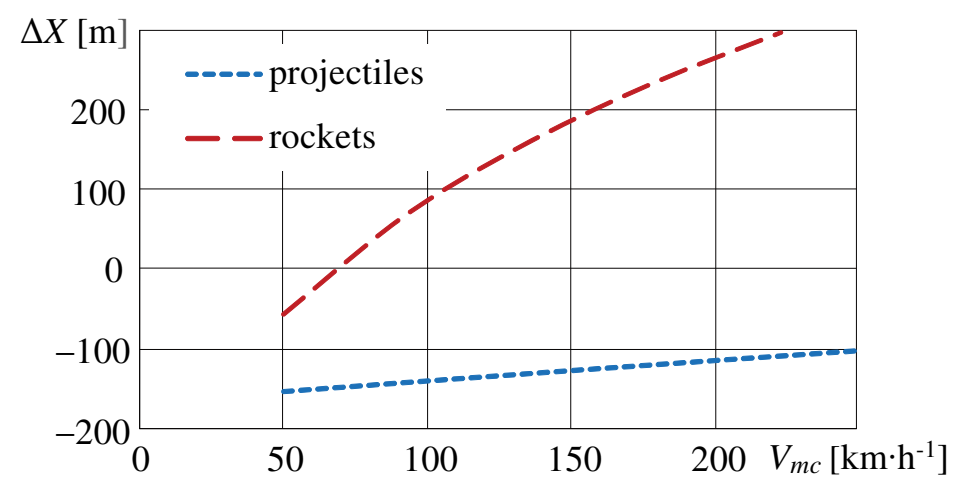

Fig. 8 Dependence of maximum deviation of incidence points of projectiles and rockets in the bursts by distance $\Delta X$ on velocity of moving carrier $V_{m c}$

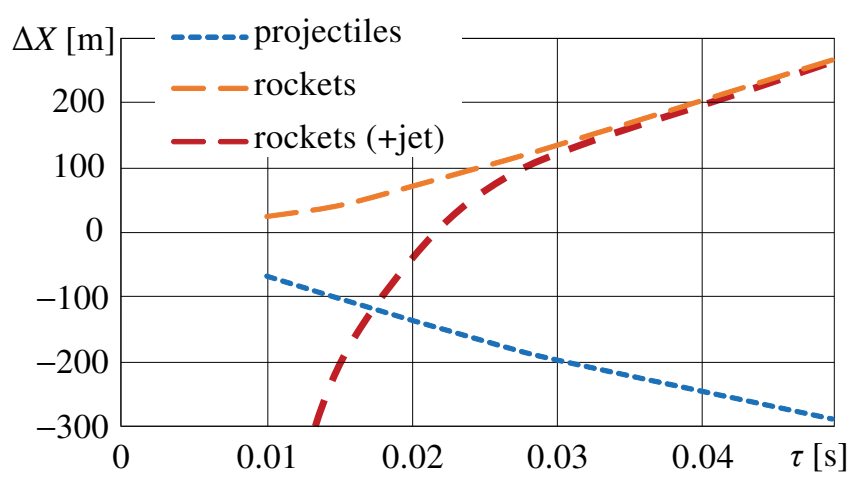

Fig. 9 Dependence of maximum deviation in range $\Delta X$ of incidence points of projectiles and rockets in the bursts on the interval between shots (launches)

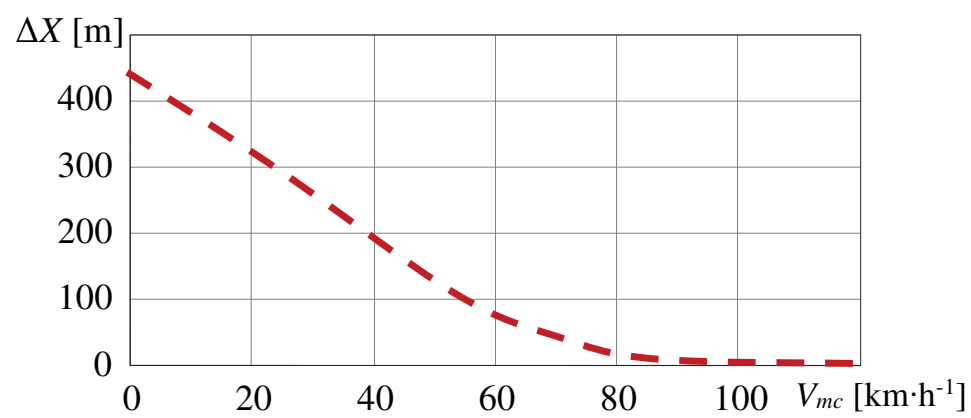

Fig. 10 Deviation in range $\Delta X$ of incidence points of rockets in the bursts under the influence of helicopter rotor 


\section{Conclusion}

The article gives a description and sets out the working principles of the software complex for modeling and three-dimensional dynamic visualization of shooting from a gun armament from a moving carrier, taking into account the terrain. The software package is a tool for studying the influence of various factors on the dispersion of projectiles and rockets at shot from a moving carrier.

The mathematical model takes into account the mutual influence of the system "helicopter - rocket-gun armament", the influence of the air flow from the helicopter rotor on the initial part of the trajectory and the mutual influence of the jet engine stream during rocket launches. The article presents some examples of research on the dispersion of projectiles and rockets in various shooting modes from a helicopter using the developed mathematical model. It is shown that the dynamics of a moving carrier has a significant and multidirectional effect on the dispersion of projectiles and rockets in serial firing. Deviation of projectiles bursts always occurs in the direction of decreasing the firing range. Rockets deviation during serial firing depends on the speed of the helicopter: for helicopter speeds less than $75 \mathrm{~km} \cdot \mathrm{h}^{-1}$, rockets deviation is in the direction of decreasing range; otherwise, it is in the direction of increasing range. The studies also show that the air flow from the main rotor has an effect on the trajectory of rockets at helicopter speed less than $80 \mathrm{~km} \cdot \mathrm{h}^{-1}$.

\section{Acknowledgment}

The reported study was funded by RFBR, project number 20-01-00072\20.

\section{References}

[1] Virtual Battlespace 3 [online]. Bohemia Interactive [viewed 2020-03-16]. Available from: https://bisimulations.com/virtual-battlespace-3

[2] COIRO, D.P., DE MARCO, A. and NICOLOSI, F. A 6DOF Flight Simulation Environment for General Aviation Aircraft with Control Loading Reproduction. In Proceedings of the AIAA Modelling and Simulation Technologies Conference and Exhibit. Hilton Head: AIAA, 2007. DOI 10.2514/6.2007-6364.

[3] CHEBOTAREV, A.S., KUDRYASHOV, V.N. and GUDZ, G.F. OKB MEI: APK Virtual Landfill (in Russian) [online]. National Defense. [viewed 2020-03-16]. Available from: http://www.oborona.ru/includes/periodics/defense/2011/0712/ 13056893/detail.shtml

[4] BRAVERMAN, A.S., GRODKO, L.N., LEYKAND, M.A., MIL, M.L. and NEKRASOV, A.V. Helicopters. Calculation and Design. Vol. 1. Aerodynamics. Washington DC: NASA, 1967. 489 p.

[5] KOZLOV, V.V. and RAMODANOV, S.M. The Motion of a Variable Body in an Ideal Fluid. Journal of Applied Mathematics and Mechanics, 2001, vol. 65, no. 4, p. 579-587. DOI 10.1016/S0021-8928(01)00063-6.

[6] KOROLEV, S.A., RUSYAK, I.G., TENENEV, V.A., VAGIN, A.V. and BELOBORODOV, M.N. Investigating the Influence of Dynamic Characteristics of a Moving Carrier on the Accuracy of Shooting. Intelligent Systems in Manufacturing, 2018, vol. 6, no. 3. p. 103-109. DOI 10.22213/2410-9304-20183-103-109. 
[7] HAIRER, E., WANNER, G. and NORSETT, S.P. Solving Ordinary Differential Equations. Berlin: Springer, 1993. 528 p. ISBN 978-3-540-78862-1.

[8] KOROLEV, S.A., RUSYAK, I.G. and SUFIYANOV, V.G. Method for Calculating the Trajectory of Projectiles and Rockets Shooting from Moving Carrier. Intelligent Systems in Manufacturing, 2016, vol. 14, no. 4, p. 13-18. DOI 10.22213/2410-9304-2016-4-13-18.

[9] RUSYAK, I.G., SUFIYANOV, V.G., KOROLEV, S.A. and ERMOLAEV, M. Software Complex for Simulation of Internal and External Ballistics of Artillery Shot. In Proceedings of the International Conference on Military Technologies. Brno: IEEE, 2015, p. 9-17. DOI 10.1109/MILTECHS.2015.7153682.

[10] KOROLEV, S.A. and KARSKANOV, S.A. Mathematical Simulation of Supersonic Airflow around the Rotary Body. Vestnik Udmurtskogo Universiteta. Matematika. Mekhanika. Komp'yuternye Nauki, 2014, no. 3, p. 123-133. DOI 10.20537/vm140311.

[11] KOROLEV, S.A., LIPANOV, A.M. and RUSYAK, I.G. On the Issue of Accuracy of the Solution of the Direct Problem of External Ballistics. Tomsk State University Journal of Mathematics and Mechanics, 2017, no. 47, p. 63-74. DOI 10.17223/19988621/47/7.

[12] KOROLEV, S.A., RUSYAK, I.G. and SUFIYANOV, V.G. Research the Influence of Disturbing Factors on the Trajectory of Projectiles and Rockets Shooting from Moving Carrier. Izvestiya Tula State University, 2017, no. 11-3, p. 23-33. ISSN 2071-6168.

[13] BRAVERMAN, A.S. and VAJNTRUB, A.P. The Dynamics of the Helicopter. Limit Flight Modes (in Russian). Moscow: Mashinostroenie, 1988. 280 p. ISBN 5-217-00108-9.

[14] LIPANOV, A.M., RUSYAK, I.G. and KOROLEV, S.A. Influence of Jet Engine Stream Impact on Rocket Movement during Salvo Launches from a Moving Carrier (in Russian). In Proceedings of the Intra-Chamber Processes and Burning in Solid Fuel Installations and Barrel Systems. Moscow: Russian Academy of Sciences, 2017, p. 161-167. ISBN 978-5-89238-111-6.

[15] NeoAxis 3D Engine [online]. [viewed 2020-03-16]. Available from: http://www.neoaxis.com 